\title{
Designer DNA Hydrogels Stimulate 3D Cell Invasion by Enhanced Receptor Expression and Membrane Endocytosis
}

\author{
Shanka Walia, ${ }^{\dagger}$ Vinod Morya, ${ }^{\dagger}$ Ankit Gangrade, Supriyo Naskar, Aditya Guduru Teja, Sameer Dalvi, \\ Prabal K Maiti, Chinmay Ghoroi, and Dhiraj Bhatia*
}

Cite This: ACS Biomater. Sci. Eng. 2021, 7, 5933-5942

Read Online

ACCESS |

Џll Metrics \& More

Article Recommendations

Supporting Information

ABSTRACT: DNA has emerged as one of the smartest biopolymers to bridge the gap between chemical science and biology to design scaffolds like hydrogels by physical entanglement or chemical bonding with remarkable properties. We present here a completely new application of DNA-based hydrogels in terms of their capacity to stimulate membrane endocytosis, leading to enhanced cell spreading and invasion for cells in ex vivo 3D spheroids models. Multiscale simulation studies along with DLS data showed that the hydrogel formation was enhanced at lower temperature and it converts to liquid with increase in temperature. DNA hydrogels induced cell spreading as observed by the increase in cellular area by almost two-fold followed by an increase in the

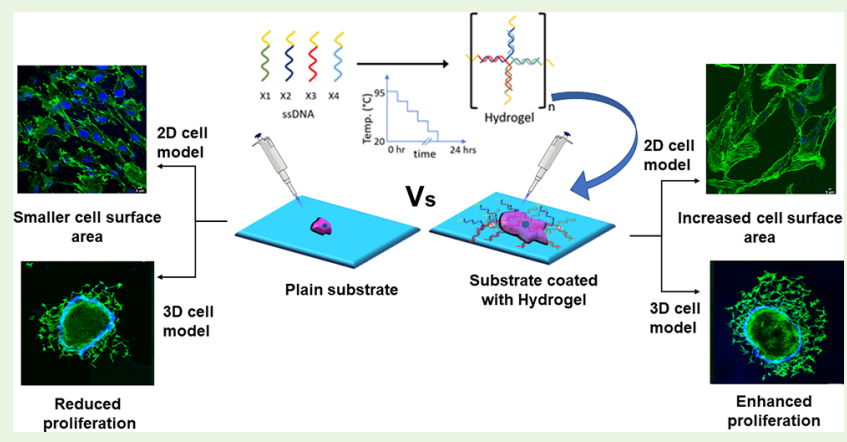
receptor expression, the endocytosis, and the $3 \mathrm{D}$ invasion potential of migrating cells. Our first results lay the foundation for upcoming diverse applications of hydrogels to probe and program various cellular and physiological processes that can have lasting applications in stem cell programming and regenerative therapeutics.

KEYWORDS: DNA hydrogels, self-assembly, cell spreading, endocytosis and 3D spheroids, cell invasion

\section{INTRODUCTION}

The extracellular matrix $(E C M)$ is a $3 \mathrm{D}$ natural scaffold produced by different proteins such as collagen, proteoglycans, fibronectin, elastin, and cell-binding glycoproteins. ${ }^{1}$ Various components of ECM are connected to form a meshlike intranet between cells, thus providing mechanical strength to the tissues. The ECM is a pool of growth factors and bioactive molecules such as fibroblasts and cytokines. The ECM provides an adherent substrate/matrix to cells by connecting through cell-binding receptors. ${ }^{2}$ The ECM controls various functions of cells, including adhesion, polarity, proliferation, differentiation, migration, gene expression, and apoptosis in vitro and in vivo. ${ }^{3}$ Because ECM is an important component for cell survival, different natural and synthetic matrices mimicking the natural ECM structure are considered as potential approaches to probe, program, and reprogram cellular behaviors in ex vivo and in vivo models. Significant research has been done in designing artificial scaffolds that exhibit the properties of ECM such as enhancing cell growth, ${ }^{4}$ mimicking natural ECM, ${ }^{5}$ maintaining cells, ${ }^{6}$ providing mechanical $^{7}$ and biological support, ${ }^{8}$ enhancing nutritional support and gaseous exchange, and excreting metabolic waste. $^{9,10}$ In this context, hydrogels with a porous network structure have been studied extensively and employed as an artificial matrix that mimics biological ECM. ${ }^{11}$ The unique properties of hydrogels include a viscoelasticity similar to that of soft tissues and the ability to retain large amounts of water, which enable efficient transport of nutrients and gases through the cells. ${ }^{12}$ Therefore, in vitro cell culturing should be supplied with such an ECM that satisfies both the biological and chemical requirements of cells. Different polymers used to synthesize hydrogels include poly(vinyl alcohol) (PVA), poly(ethylene oxide) (PEO), and poly(acrylic acid) (PAA). ${ }^{13}$ These materials are biocompatible as well as biodegradable, but on hydrolytic biodegradation, they release toxic acids that can be harmful for the cells. Also, their hydrophobic nature can lead to nonspecific adsorption of proteins, thus resulting in uncontrolled and unwanted cell interactions. ${ }^{13,14}$ Thus, to overcome these drawbacks, researchers have used biologically derived materials such as hyaluronic acid, elastin, and collagen as the cell matrix. However, because of their inherent bioactivity, reduced stiffness, tedious synthesis protocols, and variability when synthesized in batches, they were difficult to control and thus can affect immune responses when implanted. $^{15,16}$

Received: August 24, 2021

Accepted: November 22, 2021

Published: December 2, 2021

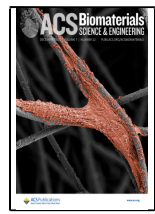


Scheme 1. Schematic Representation of DNA Hydrogel Synthesis Using ssDNA Annealed at $20-95{ }^{\circ} \mathrm{C}$ for $8 \mathrm{~h}$ and Their Application in 2D and 3D In Vitro Cell Spreading and Growth

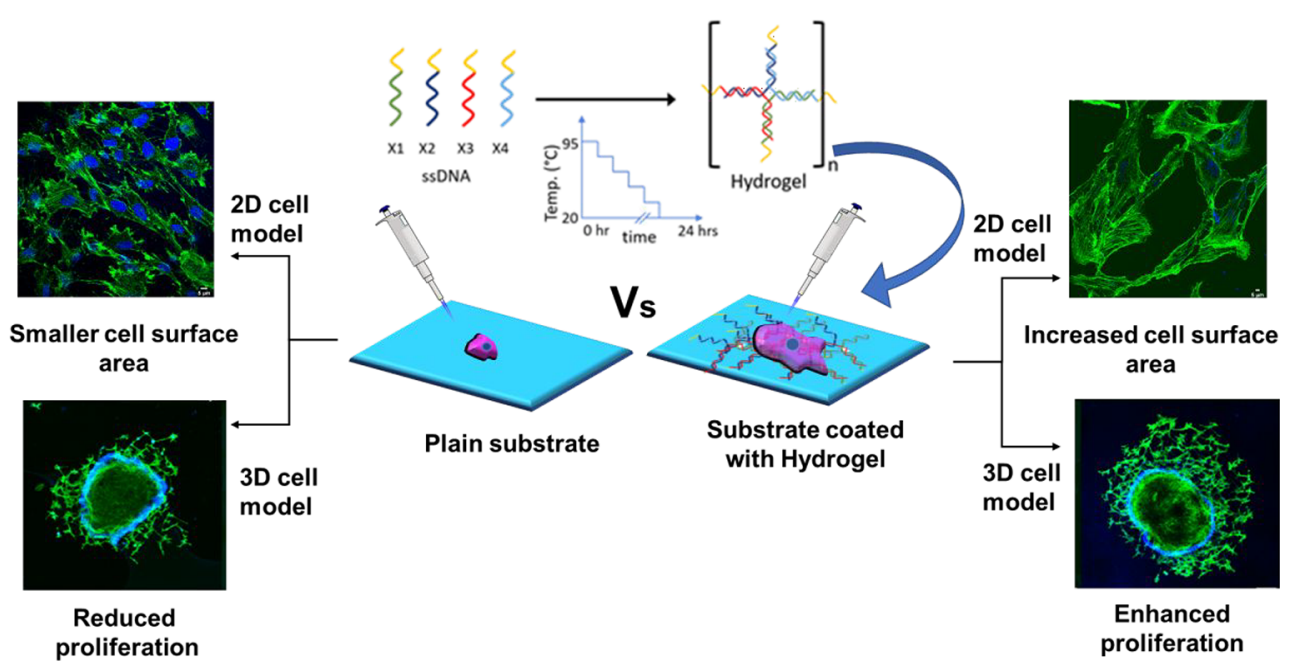

(a)

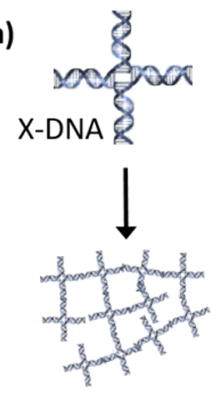

HGX (b)

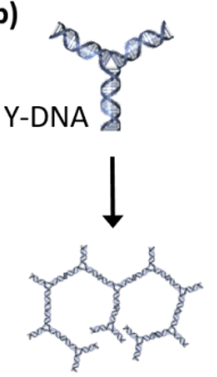

HGY (c)

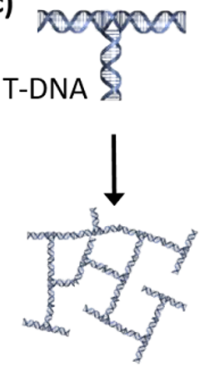

HGT (d)

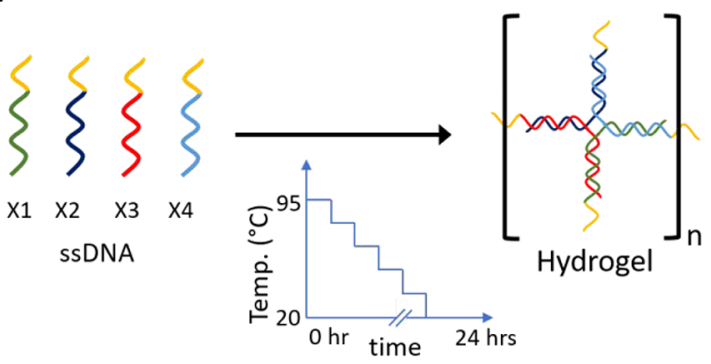

(e)

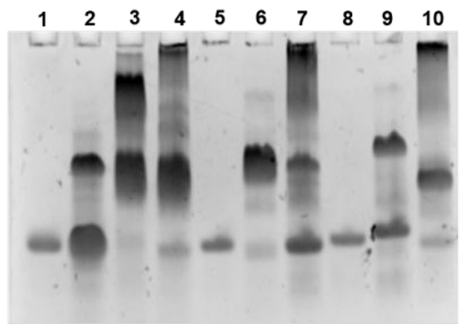

(f)

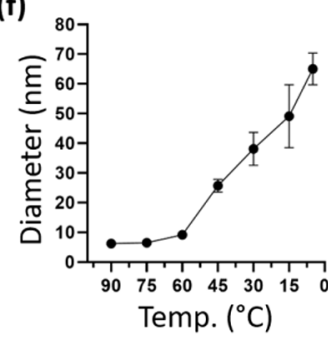

(G)
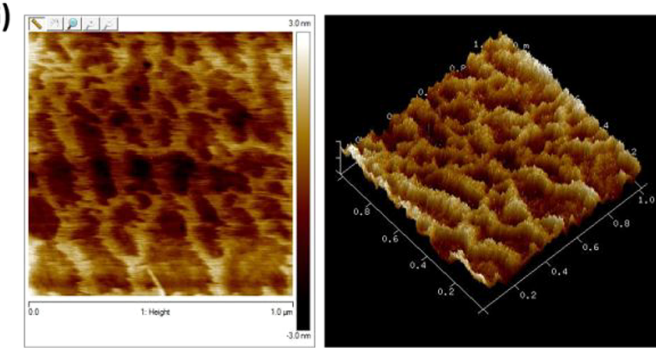

(h)

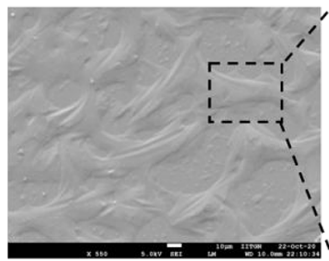

$10 \mu \mathrm{m}$

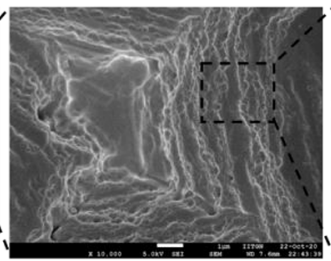

$1 \mu \mathrm{m}$

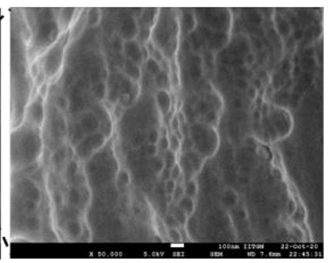

$100 \mathrm{~nm}$

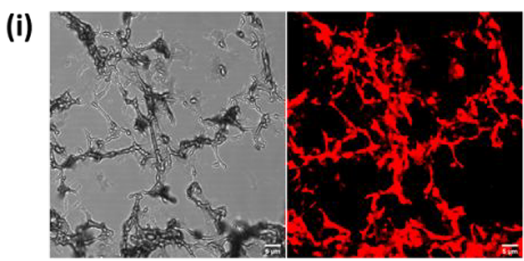

Bright Field
470/560-595

Figure 1. Synthesis and characterization of DNA hydrogels. Schematic representation of (a) X, (b) Y, and (c) T-shaped monomers and their corresponding hydrogels. (d) Hydrogel formation by self-annealing of ssDNA monomers. (e) Electromobility shift assay to verify self-assembly of the DNA hydrogels: lane 1, X1; lane 2, X1 + X2; lane 3, X1 + X2 + X3; lane 4, X1 + X2 + X3 + X4 (HGX); lane 5, Y1; lane 6, Y1 + Y2; lane 7, Y1 + $\mathrm{Y} 2$ + Y3 (HGY); lane 8, T1; lane 9, T1 + T2; lane 10, T1 + T2 + T3 (HGT). (f) Temperature-dependent formation of HGX confirmed by DLS, where increasing diameter shows the building of a higher-molecular-weight structure. (g) AFM images of HGX in 2D and 3D planes (height gradient bar $-3 \mathrm{~nm}$ to $+3 \mathrm{~nm}$ ). (h) FE-SEM micrograph of HGX showing the morphology at $10 \mu \mathrm{m}, 1 \mu \mathrm{m}$, and $100 \mathrm{~nm}$ scale. (i) Confocal images of DOX intercalated in the HGX matrix (scale bar: $5 \mu \mathrm{m}$ ).

Synthetic DNA strands can self-assemble by Watson-Crick base pairing in a meshlike structure which can swell upon addition of water, called hydrogel. ${ }^{17,18}$ DNA hydrogels have showed remarkable properties such as mechanical stability, biocompatibility, hydrophilicity, self-healing ability, precise base pairing leading to uniformity in batches. ${ }^{19,20}$ The hydrophilic nature enhances its gelling property on binding with water molecules. Because of these characteristics, DNA 

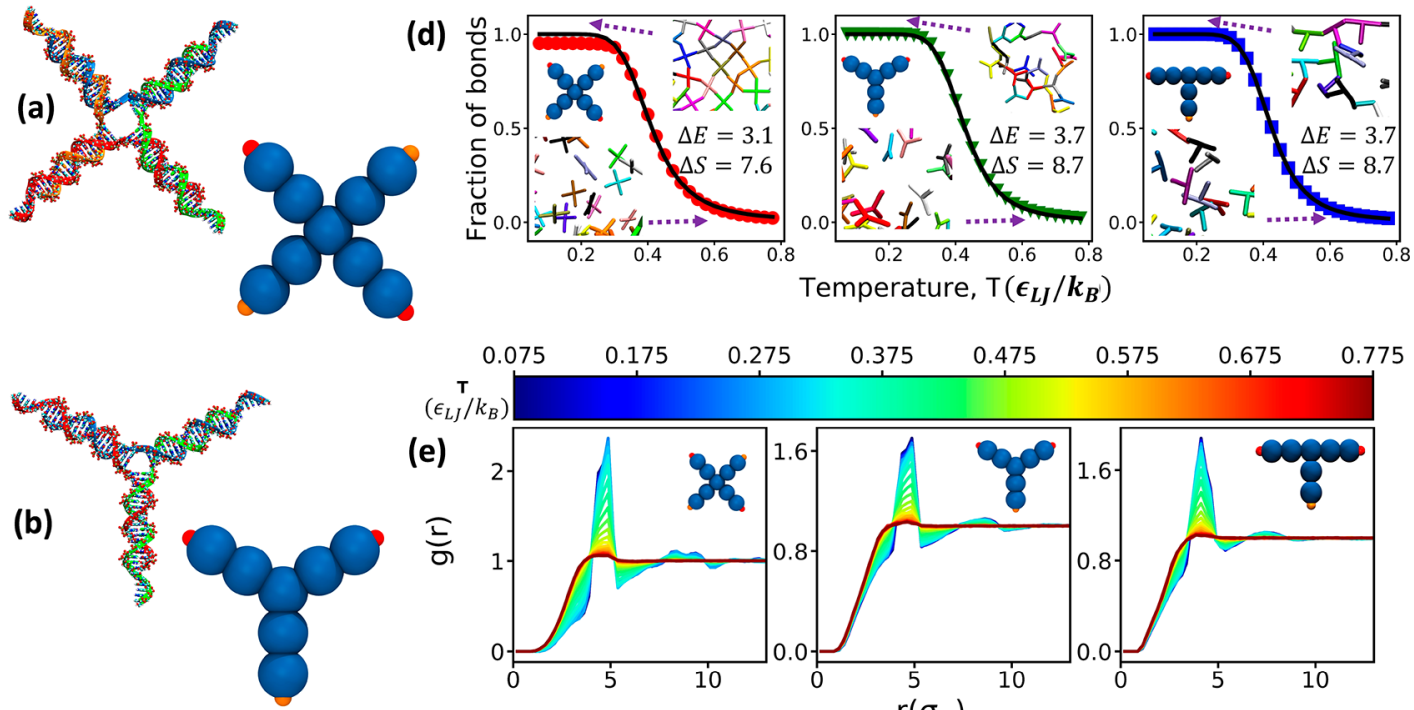

$\begin{array}{llll}0.275 & 0.375 & 0.475 & 0.575\end{array}$

\subsection{5}

0.775
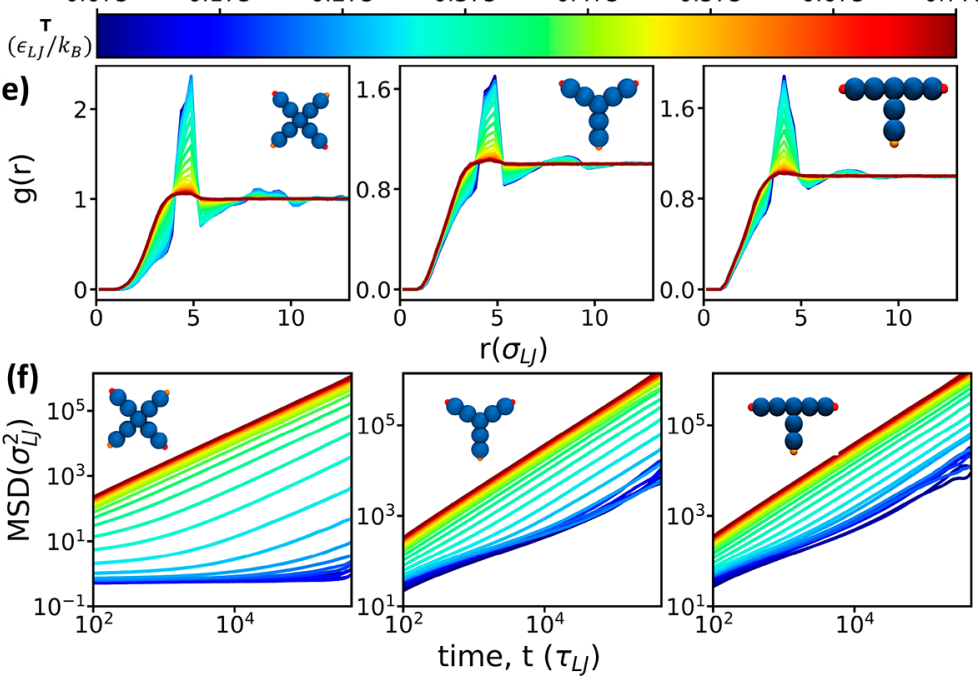

Figure 2. Numerical coarse-grained molecular dynamics simulation study of DNA gelation. (a-c) Atomistic and coarse-grained models of each nanostar. (a) X-shaped DNA nanostar. (b) Y-shaped DNA nanostar. (c) T-shaped DNA nanostar. (d) Snapshots of each system and fraction of connected nanostar pairs at different temperatures. Fraction of bonds is determined by calculating the total number of connected nanostar pairs and then dividing it by number of nanostars present in the system. The points represent simulation results, and the straight line is the fitting of a twostate model. The fitting parameters are also given in the graph. (e) Radial distribution of the function of the central bead of each nanostar system at different temperatures. (f) Mean-squared displacement (MSD) of the central bead of each nanostar system at different temperatures.

hydrogels have huge potential in cell biology applications, as these can be precisely engineered to form scaffolds that can easily control cell-cell and cell-substrate interactions. ${ }^{3,20}$ Such DNA-based scaffolds mimicking natural ECM can bridge the gap between $2 \mathrm{D}$ in vitro cell culture and in vivo animal models by reducing the natural complexity. Using the previously established designs of DNA-based hydrogels with some modifications, we showed here completely new applications of DNA hydrogels. We have synthesized and characterized DNA hydrogels using single-stranded DNA (ssDNA) by self-annealing method (Scheme 1). These hydrogels were used to understand their role in cell attachment, spreading and growth, etc. We observe that not only do these scaffolds act as cushions for cells to attache but they also stimulate cell spreading and lipid-mediated endocytosis ultimately leading to enhanced invasion of cells in 3D matrix. This study will help to improve tissue regeneration applications that are highly dependent on the adhesive property of hydrogels, where transplanted cells are mostly anchorage dependent. Most of the hydrogels lack these structural modularity properties; unlike DNA hydrogels, we cannot control the structure of monomers in other hydrogels while keeping the basic chemical properties the same. In the case of DNA hydrogel, one can choose different DNA geometries as the monomer unit, such as a three-way junction, ${ }^{21,22}$ a four-way junction, ${ }^{21}$ long single-stranded $\mathrm{DNA}^{23}$ a tetrahedron ${ }^{24}$, etc., with different sizes. This will change only the physical properties of the hydrogel, not the chemical properties. DNA hydrogels with optimized adhesive properties thus might help in the improved therapeutic response of such transplants.

\section{RESULTS AND DISCUSSIONS}

2.1. Synthesis and Characterization of DNA Hydrogels. Different types of DNA hydrogels were assembled for motifs modified from previously published results. ${ }^{21}$ The Xshaped monomer is a four-way junction DNA consisting of X1, $\mathrm{X} 2, \mathrm{X} 3$, and $\mathrm{X} 4$ oligonucleotide fragments and the resultant hydrogel was named HGX (Figure la and Table S1). Similarly, $\mathrm{Y}$ - and $\mathrm{T}$-shaped monomers were three-way junction DNA made of $\mathrm{Y} 1, \mathrm{Y} 2, \mathrm{Y} 3$ and $\mathrm{T} 1, \mathrm{~T} 2$, and $\mathrm{T} 3$, and the resultant hydrogels were named HGY and HGT, respectively (Figure $1 \mathrm{~b}, \mathrm{c})$. We modified the earlier reported oligonucleotide sequences to ensure higher thermal stability by increasing the $\mathrm{G}-\mathrm{C}$ content in the sticky ends. Both four-way junction and three-way junction DNA hydrogels were prepared by the selfannealing method (Figure 1d). To determine whether we achieve the self-assembled $\mathrm{X}-, \mathrm{Y}-$, and T-shaped DNA hydrogels, we performed an electromobility shift assay (EMSA) to confirm the stepwise assembly of DNA nanostructures (Figure 1e). The mobility of DNA strands decreased as each ssDNA, X1, X2, X3, and X4, was consequently added for a four-way junction DNA hydrogel, and a distinct band shift was observed, reflecting the effectiveness of the DNA assembly. Similar behavior was observed for the other two types, for three-way junction 
hydrogels of $\mathrm{Y} 1, \mathrm{Y} 2$, and $\mathrm{Y} 3$ and $\mathrm{T} 1, \mathrm{~T} 2$, and $\mathrm{T} 3$. By comparing the mobility of bands in distinct lanes, we concluded that the four-way and three-way junction DNA nanostructures hybridized together to form cross-linked DNA hydrogels, where a band with lower mobility corresponds to a highermolecular-weight structure. The temperature-dependent formation of higher-order structure was also confirmed by DLS, where the increase in the size of the structures was observed with a corresponding decrease in temperature (Figure 1f).

The morphological characterization of the superstructures formed was performed using AFM, FE-SEM, and confocal microscopy. Atomic force microscopy with tapping mode was performed on the DNA hydrogels, which confirmed the porous and network-like structure of hydrogel (Figure $1 \mathrm{~g}$ ). In the lyophilized state, FE-SEM images showed highly branched and cross-linked structures of DNA hydrogel (Figure 1h). Confocal images obtained in bright-field as well as in visible region showed densely interconnected structures of DNA hydrogel (Figure 1i). These characterization studies clearly demonstrated successful formation of DNA hydrogels by a selfassembly process with distinct characteristics of gel-like material.

2.2. Multiscale Modeling and Molecular Dynamics Simulation. The initial model of all the DNA nanostars (Xshaped, Y-shaped, and T-shaped) was built using the Nucleic Acid Builder module of AMBERTOOLs according to the experimental sequence design (Figure $2 \mathrm{a}-\mathrm{c}$ ). Model building and simulation details can be found in the Materials and Methods. Instantaneous snapshots of each hydrogel system at two extreme temperatures [very high $\left(T^{*} \sim 0.775\right)$ and very low $\left.\left(T^{*} \sim 0.075\right)\right]$ are shown in the inset of Figure $2 \mathrm{~d}$. From the snapshots, it is evident that at high temperature there is no structural ordering present in the system and the systems behave like unstructured fluid. However, as the temperature is lowered, the nanostars start to associate with one another through the complementary sticky end interaction and form a polymeric network. This polymeric network spans through the simulation box, cages the dynamics of the system, and gives its adhesive viscous behavior. To quantify such a polymeric network structure, we have calculated the number of connected nanostar pairs. We define that two nanostars to be connected when distance between the sticky ends is $\leq 0.5$ $\sigma_{\mathrm{LJ}}$. We divide the total number of connected bonds with the number of nanostars present in the system to get the fraction of bonds (Figure $2 \mathrm{~d}$ ). The fraction of bonds $(f)$ is then fitted with a two-state model as follows:

$$
f=1-\frac{1}{1+e^{(\Delta E-T \Delta S) / T}}
$$

Where $T$ is the temperature, $\Delta E$ is the change in enthalpy, and $\Delta S$ is the entropy change upon the phase transition. In the two-state model, the system goes from a fully bonded state to a fully unbonded state as a function of temperature. Such a phase transition is mediated by free energy change in the system. When the system forms a polymeric network, it loses a significant amount of entropy, which is compensated by the enthalpic gain due attraction between sticky ends. We find that the change in entropic is almost double to that of change in enthalpy as the system undergoes phase transition. The large entropic change originates from the localization of the nanostars in a small confining region when bonded compared to the unbonded state, where they have complete orientation and translation freedom. The contribution of enthalpic change is associated with the interaction of the complementary sticky ends. To understand the microscopic structure of the gel phase, we have computed the radial distribution function (RDF or $g(r)$ ) of the central beads for each nanostar system. The force-field parameters of the simulation like mass $\left(m_{\mathrm{LJ}}\right)$, length $\left(\sigma_{\mathrm{LJ}}\right)$, energy $\left(\epsilon_{\mathrm{LJ}}\right)$, and the Boltzmann constant $\left(K_{\mathrm{B}}\right)$ are expressed in reduced Lennard-Jones $(\mathrm{LJ})$ unit. The RDF is calculated using the following definition

$$
\mathrm{RDF}=\frac{1}{4 \pi r^{2} \rho N} \sum_{i=1}^{N} \sum_{j \neq i}^{N}\left\langle\delta\left|r_{i j}-r\right|\right\rangle
$$

Where $\rho$ is the average number density of the systems, is the total number of nanostars, and $\langle\cdots\rangle$ denotes ensemble average. The double sum counts the total number of pairs at a distance $r{ }^{25}$ The RDF for each system at different temperatures is plotted in Figure 2d. At high temperatures, RDFs do not show any well-defined peak, indicating that the nanostar mixture behaves like an unstructured fluid at high $T$. As the temperature is lowered, well-defined peaks in the RDF emerge, and it becomes more prominent at very low temperatures. These peaks in the RDF imply the growth of the polymeric network at low temperatures. The peak positions depend on the way two nanostars orient and pair with each other. This emergence of the network structures in the gel phase also arrest the overall dynamics of the systems. To probe the dynamics, we have calculated the mean-squared displacement (MSD) of the central bead of each nanostar. The MSD of each system is plotted in Figure $2 \mathrm{f}$. At high $T$, the MSD changes linearly with time, which indicates the dynamics of the structures are diffusive and more like liquid. With the lowering of temperature, the dynamics of each nanostar slowed down because of the formation of the bonds with each other, and MSD vs time deviates from their linear behavior. For the range of temperatures where MSD show linear behavior, we have calculated the diffusion constants of nanostars from the slope of the MSD vs $t$ and plotted those as a function of temperature in Figure S2. With decreasing temperature, we find that the diffusion constant of each system is decreasing. When the system forms a percolating network, the dynamics freezes, and the diffusion constant can longer be calculated from the MSD curve. We also find that the diffusion constant of X-shaped nanostar systems is slightly lower compared to Y-shaped and $\mathrm{T}$-shaped nanostar systems. The volume fraction of $\mathrm{X}$-shaped nanostar systems is higher compared to the other two nanostar systems, which reduces the available phase space volume to diffuse, and this is reflected in the overall diffusion of the system.

2.3. DNA Hydrogels Enhance Membrane Receptor Expression and Cell Spreading. Adhesion of cells to one another or extracellular matrix (ECM) plays an important role in cell communication and regulation. ${ }^{26-29}$ Cell adhesion stimulates signals for cell differentiation, cell migration, and the survival of cells. To check if DNA hydrogels had any impact on membrane receptors and cellular anchoring via focal adhesions to these scaffolds, we studied the membrane expressions of receptors and cell spread by area calculations. Increases in cell area with and without DNA hydrogels were investigated to check their role in cell spreading. Actin filaments present along the edges of cells resulted in continuous adhesion by distribution and reorganization, thus helping in cell spreading. $^{30}$ 
When the actin cytoskeleton was stained and visualized using phalloidin green, the results suggested that on culturing RPE1 cells to these hydrogels, such as HGX, HGY, and HGT, cells were nicely adhered and spread on these DNA scaffolds as compared to the control sample (Figure 3 and Figure S2). (a)

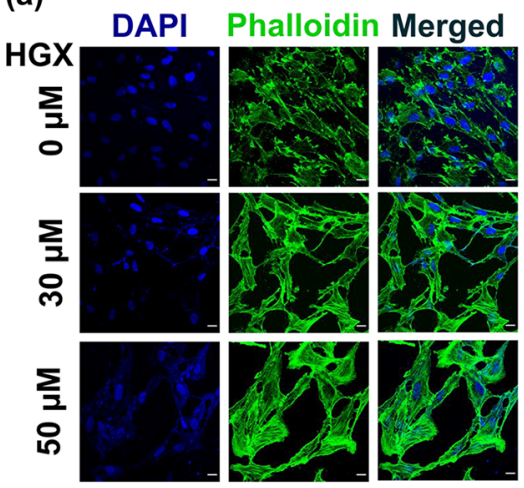

(b)

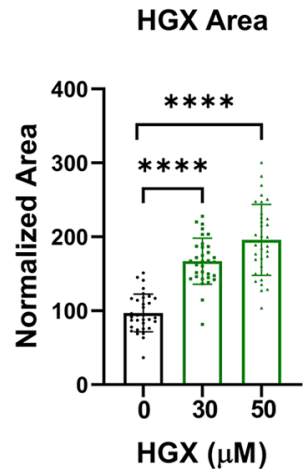

Figure 3. Effect of DNA hydrogels on cellular spreading. (a) Confocal microscopic images of RPE 1 cells incubated on the carpet of 30 and $50 \mu \mathrm{M}$ HGX for $24 \mathrm{~h}$, at $37{ }^{\circ} \mathrm{C}$. Scale bar is $10 \mu \mathrm{m}$. (b) Quantification of normalized cell area w.r.t concentration. ****, statistically significant value of $p<0.0001$ (one-way ordinary ANOVA).

From the quantification data it was found that there was significant increase in cell area in the presence of DNA hydrogel as compared to control samples (Figure 3b). Also, the actin cytoskeleton was reorganized in cells plated on hydrogels

(a)

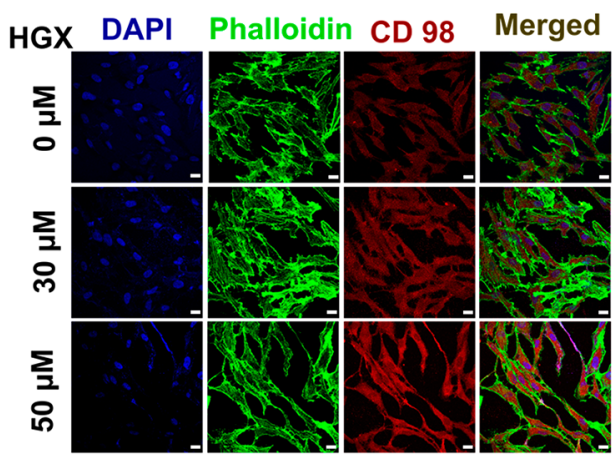

(c)
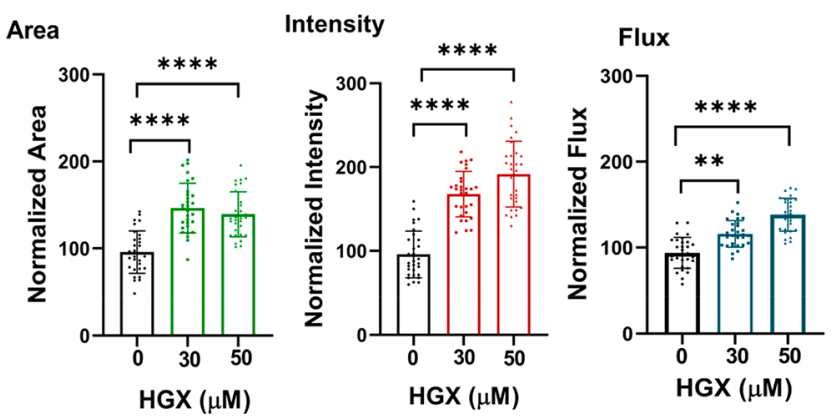

as seen by the visible change in their intracellular arrangement (Figure 3a). We further noticed that the increase in cell area was concentration dependent in DNA hydrogels, showing a two-times increase in cell area in the case of DNA hydrogels as compared to control samples (Figure 3 and Figure S2). The increase in cell area was comparable to that of the positive control, i.e., poly-L-lysine. Because all of the hydrogels (HGX, HGY, and HGT) show similar results but the HGX demonstrated the best of the spreading results, all of the subsequent studies were done with HGX. There are no chemical growth factors involved, and thus the increase in the cellular spreading could be due to either physical interactions or binding via receptors like ALBRs (anionic ligand binding receptors). The increase in the cell spreading may be due to the crawling of cells over the favorable hydrogel matrix. The hydrogel's networklike structure encourages cells to hold the immobilized fibers, which generates strains in the membrane and causes the expansion and growth of the membrane.

Did the increase in cell area on DNA hydrogels affect plasma membrane receptor expression and influence downstream endocytic pathways? To probe these questions, we performed immunofluorescence staining of the plasma membrane fractions of the membrane receptors like CD98 and CD147. These are the ectopic receptors present on the plasma membrane and are known to change their expression in response to ECM and disease conditions. ${ }^{31-34} \mathrm{We}$ recorded high signal intensity for CD98 and CD147 on the HGX-coated surface compared to an uncoated surface upon immunofluorescence imaging. To further confirm whether these receptors are overexpressed or if the high fluorescence intensity on the (b)

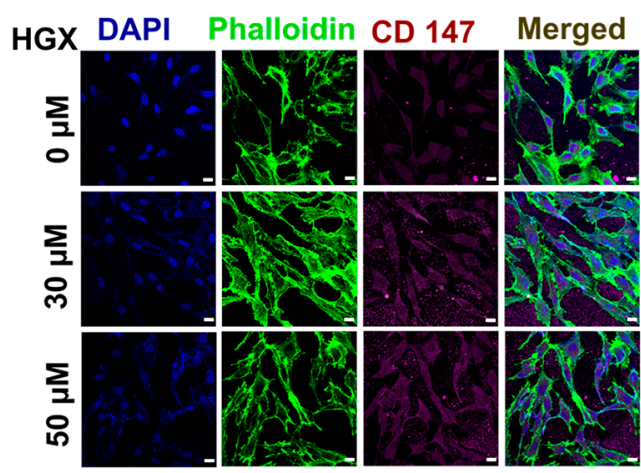

(d)
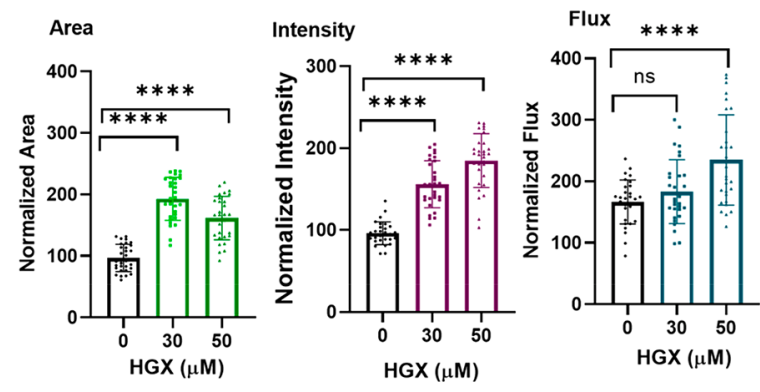

Figure 4. Confocal microscopic images and quantification of Immunofluorescence assay RPE1 cells with and without increasing concentration of HGX incubation (a) with CD98 (red) and (b) with CD147 (red), respectively. In both cases, cellular cytoskeleton was stained with phalloidin green (green). Scale bar is $10 \mu \mathrm{m}$. (c, d) Cell area, total intensity, and flux (intensity per unit area) for CD98 and CD147, respectively. For quantification, six independent images were captured from each sample, and five cells per image were quantified with the total sum of $n=30$ using ImageJ software. $* * * *$, statistically significant value of $p<0.0001$; ** statistically significant value of $p<0.0073$; and ns, no significant difference in the data sets (one-way ordinary ANOVA). 

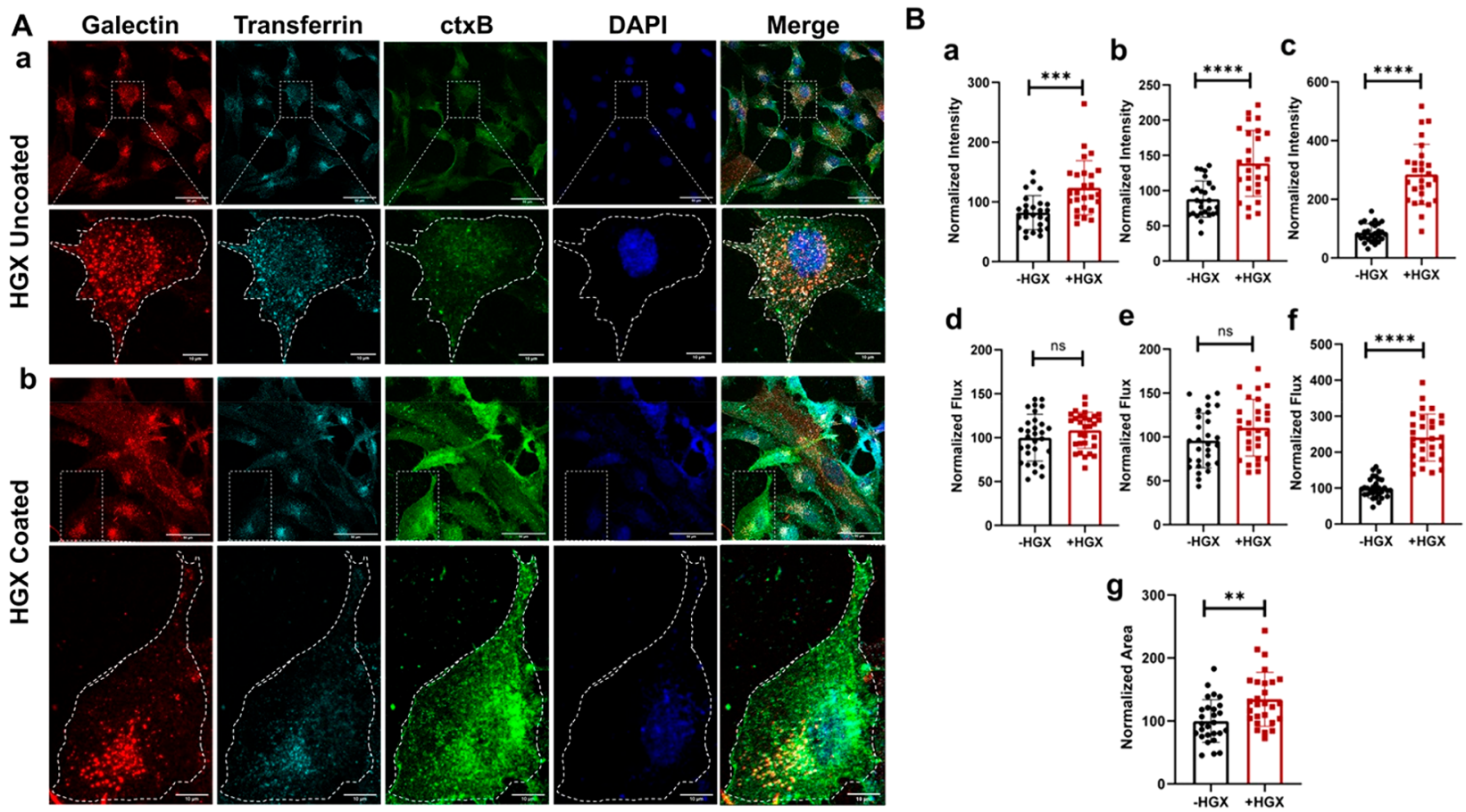

Figure 5. DNA HGX stimulates endocytosis of lipid-binding cargos. (A) Confocal images of endocytic probes such as galectin, transferrin, and cholera toxin B (CTxB) internalized by RPE1 cells grown over (a) HGX uncoated and (b) HGX-coated glass surface. Scale bar represents $50 \mu \mathrm{m}$ in zoom out (top row) and $10 \mu \mathrm{m}$ in zoomed in (bottom row) images of the panel (Aa) and (Ab). (B) Bar diagrams showing fluorescence normalized intensity and normalized flux of ( $a, d)$ galectin, (b, e) transferrin, and (c, f) CTxB. (g) Showing representative normalized cell area. For quantification, six independent images were captured from each sample, and five cells per image were quantified with the total sum of $n=30$ using ImageJ software. The statistic difference was performed using unpaired $t$ test and indicated by an asterisk $(*)$ in the normalized intensity plot, where $* * * *, p<0.0001 ; * * *, p<0.0003 ; * *, p=0.002$; and ns, no significant difference in the data sets.

HGX-coated surface was recorded because of enhanced cell volume, we calculated the flux, the fluorescence intensity per unit area. As shown in Figure $4 \mathrm{c}$, d, the flux was also significantly increased, indicating the enhanced expression of CD98 and CD147 on DNA HGX-coated surfaces compared to uncoated surfaces.

In the case of CD98, cell area was increased up to 1.5 times on treatment with hydrogel, whereas fluorescence intensity and flux were enhanced up to 1.8 and 1.4 times, respectively (Figure 4a, c. The change in the area, intensity, and flux in CD147 was $\sim 1.83,1.67$, and 1.5 times as observed in CD147 (Figure $4 b, d$. Increased cell area might have improved the nutritional requirement of cells, causing higher intracellular trafficking of treated proteins. Also, the graph plotted for flux (fluorescence intensity per unit area) showed greater protein levels in the case of HGX as compared to control. However, the change in cellular uptake of endocytic cargos is not dependent on the concentration of HGX. It was noticed that there was not much change in the cellular uptake of the receptor protein w.r.t. to the concentration of HGX, although HGX treatment resulted in an increased surface area of cells. However, an increase in the concentration of HGX from 30 to $50 \mu \mathrm{M}$ showed minimal effect on the cell surface area resulting in minimal difference in cellular uptake of treated proteins. This suggested that CD98 and CD147 accumulation was increased in the presence of HGX. Thus, from the study, it was observed that hydrogel increased the cell area, which is related to the enhanced cell migration cell signaling and nutrient uptake.

2.4. DNA Hydrogels Stimulate Endocytosis of LipidBinding Ligands. Endocytosis is a process for internalizing outer material inside the cells and has been implicated in many fundamental cellular functions such as homeostasis, differentiation, signal transmission, cell growth, etc. ${ }^{35}$ Endocytosis can take place via multiple routes by either clathrin-mediated or clathrin-independent processes. Transferrin (Tf) and galectin-3 (Gal3) are classical ligands used to study the clathrin-mediated and clathrin-independent pathways, respectively. ${ }^{36}$ Cholera toxin B-subunit (CTxB) binds to lipid GM1 on the plasma membrane and builds tubular endocytic tubules that are scissioned by cellular machinery like dynamin or actin or endophilin. These internalized vesicles then mature into early endosomes from where $\mathrm{CTxB}$ retrogradely transports to endoplasmic reticulum via plasma membrane and Golgi bodies. $^{37}$ We compared the change in cellular internalized fractions of all the above ligands in RPE1 cells seeded over DNA HGX coated and an uncoated glass coverslip. To our observation, the cellular area has significantly increased on the DNA HGX-coated surface (Figure 3B). Because of increased cell volume on the DNA HGX-coated surface, the intracellular uptake of $\mathrm{Gal} 3$, transferrin, and $\mathrm{CTxB}$ was also significantly increased, as shown in quantification data (Figure 5A, Ba-c). The higher uptake of these ligands is in agreement with the increased cell area. The larger cell size may enhance the cell's nutritional requirement, causing a higher uptake of ligands. In contrast, the fluorescence intensity per unit area, also called flux, was plotted, and surprisingly, we recorded a nonsignificant difference in the uptake of galectin and transferrin (Figure 5Bc, d). However, a significantly high flux intensity was recorded in $\mathrm{CTxB}$ treatment (Figure $5 \mathrm{Be}$ ). This could be due to lot of protein receptors being involved in the endocytosis of $\mathrm{Tf}$ and Gal3, whereas the uptake of $\mathrm{CTxB}$ involves mostly lipids on 
(a)

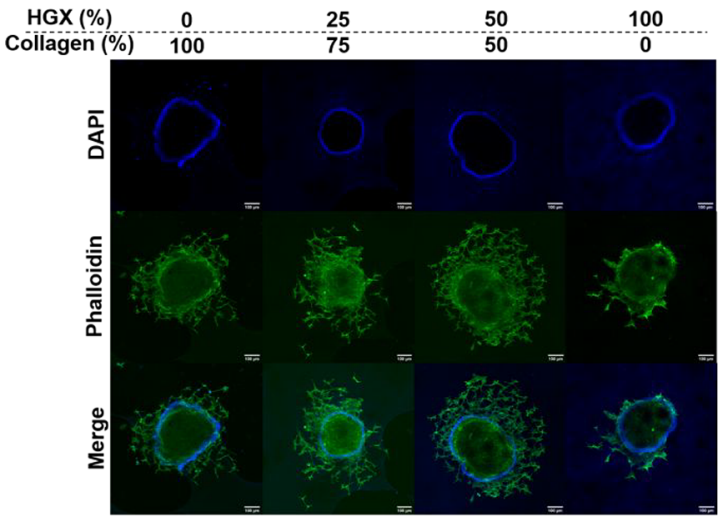

(b)

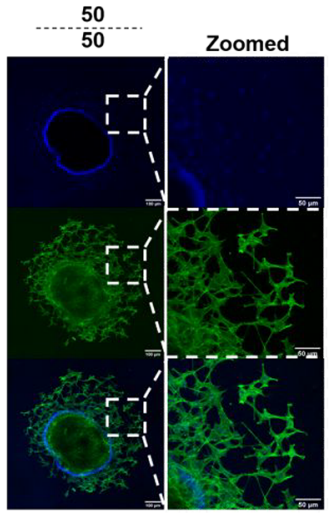

(c)

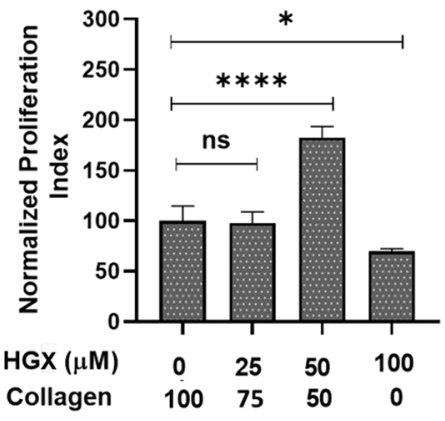

Figure 6. DNA hydrogels stimulate cellular invasion in 3D spheroid model. (a) 3D spheroids grown on different concentrations of HGX for $24 \mathrm{~h}$ showed different invasion of cells in the 3D matrix as a function of the concentration of HGX. Scale bar is $100 \mu \mathrm{m}$ for all the images. (b) Zoom image of 3D spheroid treated with $50 \mu \mathrm{M}$ of HGX with $50 \%$ collagen showing enhanced cellular invasion from the surface of the spheroid in 3D. Scale bar is $50 \mu \mathrm{m}$. (c) Quantification data of the cell invasion index show higher invasion indexes for DNA hydrogels up to a concentration of 50 $\mu \mathrm{M}$. For quantification, three independent images were captured from each sample and quantified with the total sum of $n=3$ using Image J software. $* * * *$, statistically significant value of $p<0.0001 ; *$, statistically significant value of $p=0.02$; ns, no significant difference in the data sets (one-way ordinary ANOVA).

the plasma membrane. In summary, we inferred that the DNA HGX coating enhances the cell growth and area without affecting clathrin and clathrin-independent endocytic pathways but definitely stimulating the uptake of lipid-binding cargo like $\mathrm{CTxB}$, which drives the uptake of GM1 lipids.

2.5. DNA Hydrogels Stimulate 3D Cellular Invasion. Two-dimensional (2D) cell cultures do not mimic in vivo cell growth conditions satisfactorily. The 3D spheroid model allows the development and interaction of cells with the extracellular matrix in three-dimensional environment very much similar to the in vivo conditions. ${ }^{38,39}$ We thus proceeded to evaluate the $3 \mathrm{D}$ cellular invasion behavior on DNA hydrogels using 3D culture spheroid models. Spherical spheroids made from triple-negative breast cancer cells MDA-MB-231 were transferred to 3D matrix conditions either made of pure collagen or collagen-HGX mixed HGX hybrids in different ratios and the spheroids were grown for $24 \mathrm{~h}$ to study their invasion in 3D. The results suggested that hydrogels (HGX) retained the morphology of cells (Figure 6). Confocal images along with quantification data showed not only successful but enhanced cell migration and adherence in $3 \mathrm{D}$ spheroids in the presence of hydrogels (Figure 6a, c). It was observed that on incubation with three different concentrations of 25,50 , and $100 \mu \mathrm{M} \mathrm{HGX}$, there was a differential effect in terms of increased cell invasion at $25 \mu \mathrm{M}$ HGX, whereas cell proliferation was enhanced at $50 \mu \mathrm{M}$ HGX showing a two-times increase in cell invasion (Figure 6b) and an inhibitory effect was observed at $100 \mu \mathrm{M}$ HGX.

\section{CONCLUSIONS}

We synthesized DNA hydrogels using simple, pre-established protocols using three- and four-way junctions via the selfassembly method. These DNA hydrogels when characterized showed hierarchical structural properties and striking internal morphology, confirming our DNA hydrogel predicted designs. Simulation studies along with DLS data showed the formation of a polymeric network structure of hydrogel due to the association of bonds with one another through the sticky ends, whereas these act as unstructured fluid when the temperatures are high enough. This study showed that these hydrogels are thermoresponsive hydrogels and can be used as cushions for cellular studies. Cellular studies showed that DNA hydrogels resulted only in enhanced cells adhering to these scaffolds, but this further leads to an increase in cell area as well as enhanced uptake of endocytic membrane cargos. The cellular area significantly increased on the DNA HGX-coated surface, resulting in increased cell volume and the increased expression of membrane receptors like CD98 and CD147. The larger cell size may enhance the cell's nutritional requirement, causing a higher uptake of ligands like lipids as seen by the increased uptake of lipid-binding ligands CTxB. Further, these polymeric structures gel perfectly well with scaffolds from the ECM like collagen and this hybrid composition presents a better ex vivo environment to cells, resulting in increased cell invasion in 3D spheroids. Taken together, this study establishes the launch pad of further exploring these DNA-based hydrogels either individually or in combination with other biocomponents and together, these hydrogels are poised with attractive properties and have the potential for real-world applications, including tissue engineering, drug release, cell therapy, and biosensing, to name a few.

\section{MATERIALS AND METHODS}

All oligonucleotides (Table S1) at a $0.2 \mu \mathrm{M}$ synthesis scale with desalting purification were purchased from Merck (Sigma-Aldrich). Moviol, Triton-X, loading dye, Alexa Fluor (phalloidin) 488, moviol, poly-L-lysine, membrane glycoproteins (CD98 and CD147), transferrin 488, galectin, Hoechost (DAPI), and Cholera toxin B-subunit $(\mathrm{CTxB})$, were also purchased from Merck (Sigma-Aldrich). Antibodies for CD98 (catalogue \#128202) and CD147 (catalogue \#123701) were purchased from BioLegend. Ammonium persulfate, ethidium bromide, Triton- $\mathrm{X}$, tetramethylethylenediamine (TEMED), paraformaldehyde, and cell culture dishes for adherent cells (treated surface) were procured from Himedia. Dulbecco's modified Eagle's medium (DMEM), fetal bovine serum (FBS), penicillin-streptomycin, trypsin-ethylenediaminetetraacetic acid (EDTA) (0.25\%), and Collagen1 rat tail were purchased from Gibco. Tris-acetate-EDTA (TAE), and acrylamide/bisacrylamide sol 30\% (29:1 ratio for SDS PAGE) were procured from $\mathrm{GeNei}$. Magnesium chloride, $\mathrm{NaCl}, \mathrm{KCl}$, $\mathrm{Na}_{2} \mathrm{HPO}_{4}$, and $\mathrm{KH}_{2} \mathrm{PO}_{4}$ were purchased from SRL, India, and Santa Cruez Biotech. 
4.1. Cell Culture. RPE1 cells (human retinal pigment epithelial-1 cell line) and human breast adenocarcinoma (MDA-MB-231) cells were obtained as a gift from Prof. Ludger Johannes at Curie Institut, Paris, France. RPE1 and MDA-MB-231 cells were cultured in DMEM supplemented with $10 \% \mathrm{FBS}$ and $100 \mathrm{IU} / \mathrm{mL}$ penicillin-streptomycin. For all the studies, PBS of $1 \times$ strength at $\mathrm{pH} 7.4$ was used.

4.2. Synthesis of DNA Hydrogels. Monomeric junctions of DNA ss strands were assembled from single strands (ssDNAs) and each strand had a sticky end serving as the building unit. X-shaped (X1, X2, X3, and X4), Y-shaped (Y1, Y2, and Y3), and T-shaped (T1, T2 and T3) DNA strands at a concentration of $100 \mu \mathrm{M}$ were individually added to $2 \mathrm{mM}$ bridging agent $\left(\mathrm{MgCl}_{2}\right)$. The resulting mixtures were annealed at a temperature range of $20-95{ }^{\circ} \mathrm{C}$ for $8 \mathrm{~h}$. Finally, the obtained mixtures of X-, Y-, and T-shaped DNA hydrogels were stored at $4{ }^{\circ} \mathrm{C}$ for further studies.

4.3. Characterization. 4.3.1. Electrophoretic Mobility Shift Assay. The $10 \%$ native polyacrylamide gel electrophoresis (PAGE) was prepared by using $1 \times$ Tris-acetate-EDTA (TAE) buffer. The loading sample was prepared by mixing $1-2 \mu \mathrm{L}$ of DNA samples, 5 $\mu \mathrm{L}$ of loading buffer $(5 \times)$, and $1.5 \mu \mathrm{L}$ of loading dye and kept for 3 min so that the dye could integrate with the DNA completely. The loading sample was then applied onto the lane and gel was run at $90 \mathrm{~V}$ for $90 \mathrm{~min}$ in $1 \times \mathrm{TBE}$ running buffer. The bands were stained with ethidium bromide, and then scanned using a Syngene gel documentation system.

4.3.2. Dynamic Light Scattering. The formation of a highermolecular-weight DNA hydrogel by annealing was assessed by analyzing the hydrodynamic diameter using a Malvern Panalytical Zetasizer Nano ZS instrument. Samples containing a equimolar ratio of the ssDNA oligos for respective hydrogels were analyzed in a lowvolume quartz cuvette. The hydrodynamic diameter was observed at different temperatures in decreasing order from 90 to $5{ }^{\circ} \mathrm{C}$. The temperature difference between two steps was kept at $15{ }^{\circ} \mathrm{C}$ and samples were allowed to incubate at each step for $15 \mathrm{~min}$.

4.3.3. Atomic Force Microscopy Studies. The 3D morphological characterization of synthesized DNA hydrogels was done using a Bruker Multimode 8 atomic force microscope (AFM) in tapping mode to simultaneously collect height and phase data. AFM scanning was done via the peak force tapping mode to analyze the surface thickness, height, and roughness. A triangular-shaped silicon nitride tip with a $2 \mathrm{~nm}$ radius was used for this study. The frequency was 70 $\mathrm{kHz}$ and the scan size was $1 \mu \mathrm{m} \times 1 \mu \mathrm{m}$. The hydrogel $(100 \mu \mathrm{M})$ was drop-casted on a mica sheet and freeze-dried before analysis.

4.3.4. Scanning Electron Microscopy Analysis. The porous morphology of the hydrogel was characterized using a Jeol JSM$7600 \mathrm{~F}$ field emission scanning electron microscope (FE-SEM) at an acceleration voltage of $5 \mathrm{kV}$. To prepare samples for imaging, we drop-casted and freeze-dried $10 \mu \mathrm{L}$ of hydrogel on a carbon-coated stub. A thin layer of platinum metal was sputter-coated on the lyophilized sample prior to imaging.

4.3.5. Confocal Microscopy Analysis. For confocal microscopic studies, DNA hydrogel was mixed with doxorubicin (DOX) in an equimolar ratio. An appropriate amount of sample was loaded onto glass sides and freeze-dried. The sample was imaged under a confocal microscope in bright field as well as at $560 \mathrm{~nm}$ on excitation of 488 $\mathrm{nm}$. The imaging was done using confocal laser scanning microscopy (Leica TCS SP8).

4.3.6. Simulation Studies. Conventional all-atom molecular dynamics (MD) simulation is limited to small length and time scales because of computational demand and is not suitable yet for studying gelation. So, we have used coarse-grained (CG) approach to probe the gelation of DNA nanostars. In recent years, many CG models of different resolutions have been developed to study DNA. Most of them are computationally expensive and are also too detailed, often not required to understand gelation. In this study, we have employed a minimal CG model of DNA developed by Frenkel and co-workers, based on the experimental data of Xing et al. ${ }^{40}$ The initial all-atom models of DNA nanostars are converted to a minimal CG model comprising beads and springs. In this model, the double-stranded arm of the DNA nanostars is represented by a large bead that interacts with other double-stranded parts with repulsive WCA potential. The single-stranded DNA ends of each nanostar's arm were represented by small sticky beads, which attracts other single-stranded DNA ends with a Lennard-Jones (LJ) potential of 6-12. Each arm is represented by two large beads and a small sticky bead, and they are linked to each other by a central bead. The beads were connected by harmonic bond and angle potentials. All the force-field and simulation parameters were represented by reduced $\mathrm{LJ}$ units.

After building the initial CG model, we put 250 nanostars of a particular kind into a cubic box of length $30 \sigma_{\mathrm{LJ}} \times 30 \sigma_{\mathrm{LJ}} \times 30 \sigma_{\mathrm{LJ}}$. The number density of each system was $9.25 \times 10^{-3}$. The volume fractions of Y-shaped, T-shaped, and X-shaped nanostar systems were 6.75 , 6.75 , and 9.05 , respectively. After building the initial model, we subjected each system to long MD simulation using the Langevin equation of motion. We cooled the system from a very high temperature $\left(0.775 \epsilon_{\mathrm{LJ}} / k_{\mathrm{B}}\right)$ to a very low temperature $\left(0.075 \epsilon_{\mathrm{LJ}} / k_{\mathrm{B}}\right)$ with an interval of $0.025 \epsilon_{\mathrm{LJ}} / k_{\mathrm{B}}$. At each temperature, the systems were subjected to long $\mathrm{MD}$ simulation $\left(1 \times 10^{8}\right.$ steps $)$ using an integration time step of $0.005 \delta \mathrm{t}$ in the NVT ensemble. To confirm that the systems were equilibrated at each temperature, we ensured that all the thermodynamic quantities attained steady state. The damping parameter of the Langevin dynamics is chosen to be large $(\sim 100)$ to mimic the overdamped condition.

4.4. In Vitro Cell Studies. 4.4.1. Confocal Imaging Studies. The studies were performed on $10 \mathrm{~mm}$ glass coverslips coated with/ without poly L-lysine (PLL) placed in 24-well plates. Four different concentrations of $20,30,50$, and $100 \mu \mathrm{M}$ were mounted on the coverslip and incubated at $37{ }^{\circ} \mathrm{C}$ for $30 \mathrm{~min}$. Afterward, trypsinized cells (approximately 40000 cells/well) were seeded onto hydrogeltreated coverslips and incubated for $24 \mathrm{~h}$ at $37{ }^{\circ} \mathrm{C}$ in $5 \% \mathrm{CO}_{2}$. The cells were washed with PBS and fixed using $4 \%$ PFA at $37^{\circ} \mathrm{C}$ for 15 min. The cells were washed twice with PBS and actin filament staining was done using phalloidin 488 to check cell migration in the presence of the hydrogel. Cells were first treated with Triton-X for $10 \mathrm{~min}$ at room temperature and resuspended in $500 \mu \mathrm{L}$ of phalloidin for 20 min at $37{ }^{\circ} \mathrm{C}$. After incubation, cells were washed with PBS thrice and stained with DAPI and mounted using mounting medium (moviol). All cellular fluorescent images were collected on a Leica TCS SP5 confocal microscope with a $63 \times$ oil immersion objective. A $408 \mathrm{~nm}$ argon laser was the excitation source for DAPI, whereas a $488 \mathrm{~nm}$ argon laser was used as the excitation source for phalloidin.

4.4.2. Immunofluorescence Assay. For this assay, coverslips placed in 24-well plates were coated with 30 and $50 \mu \mathrm{M}$ HGX and incubated at $37^{\circ} \mathrm{C}$ for $30 \mathrm{~min}$. Further, trypsinized cells were seeded on to these coverslips with a cell density of 40000 cells/well and incubated at 37 ${ }^{\circ} \mathrm{C}$ for $24 \mathrm{~h}$. After $24 \mathrm{~h}$, the cells were washed thrice and incubated with primary antibodies, namely, CD98 and CD147 for $30 \mathrm{~min}$ at 37 ${ }^{\circ} \mathrm{C}$. The antibodies were prepared in serum-free media in a ratio of 1:100. Fixation of cells was done using 4\% PFA by incubating for 15 min at $37^{\circ} \mathrm{C}$. Excess PFA was washed off with $1 \times$ PBS. The fixed cells were then permeabilized using $0.1 \%$ Triton-X-100 in PBS for $10 \mathrm{~min}$ at room temperature. After the removal of excess Triton-X-100, the permeabilized cells were stained with phalloidin and incubated $37^{\circ} \mathrm{C}$ for $20 \mathrm{~min}$. The removal of excess phalloidin using PBS was followed by incubation with secondary antibody prepared in $0.1 \%$ Triton-X (1:250), namely, Alexa 647 in the case of CD98 and CD147. Further, cells were incubated at room temperature in dark conditions for $2 \mathrm{~h}$. The excess of secondary antibodies was removed by washing the cells with PBS thrice. Finally, the cells were mounted in a DAPI-containing mounting media and stored at $4{ }^{\circ} \mathrm{C}$ until imaging.

4.4.3. Endocytosis Pathway Assessment in RPE1 Cells Grown over DNA HGX-Coated/Uncoated Surface. Human retinal pigment epithelial-1 cells were grown over the DNA HGX coated and uncoated glass coverslips to assess the change in cellular uptake capacity using standard endocytic probes like galectin, transferrin, and $\mathrm{CTxB}$ at a $5 \mu \mathrm{g} / \mathrm{mL}$ working concentration. The cells were treated with these probes for $15 \mathrm{~min}$ at $37{ }^{\circ} \mathrm{C}$, followed by $4 \%$ PFA fixation. The fixed coverslips were then mounted on glass slides for capturing the images at $63 \times$ resolution using a confocal laser scanning microscopy (Leica TCS SP8). The raw intensity density of 
fluorescence images was quantified using ImageJ software and normalized with respective - HGX control. The statistical difference was performed using an unpaired $t$ test and indicated by an asterisk $(*)$ in the normalized intensity plot, where $* * * *$ indicates $P<0.0001$; *** indicates $P<0.0003$; ** indicates $P=0.002$, and ns indicates no significant difference in the data sets.

4.4.4. MDA-MB 3D Model Preparation. MDA-MB-231 3D spheroids used for the cell spreading studies were prepared by the hanging drop culture method following a literature report with some modifications. ${ }^{41}$ For the preparation of hanging drops, a T25 flask with $85 \%$ cellular confluency was trypsinized using $1 \mathrm{~mL}$ trypsin. Appropriate amount of fresh complete medium was added to the trypsinized solution and mixed well to get a homogeneous suspension. Briefly, $50 \mu \mathrm{L}$ complete medium containing 40,000 cells were seeded in the form of drops over the inner surface of the Petri dish lid. Around $30 \mathrm{~mL}$ PBS was added to the Petri dish base for providing moisture environment for spheroid growth. The Petri dish lid containing cell droplets was placed upside down covering the base of the Petri dish. The cells were allowed to grow until spheroid formation was visibly observed as the formation of cell aggregates. Spheroid formation was confirmed by visualizing cell aggregates under an upright bright-field optical microscope. Once the spheroids were formed, these were transferred using collagen $(3 \mathrm{mg} / \mathrm{mL})$ and media in a ratio of 3:1 to 24 well plates containing 25,50 , and $100 \mu \mathrm{M}$ HGX in required wells and incubate for $\sim 40 \mathrm{~min}$ at $37{ }^{\circ} \mathrm{C}$. Finally, the spheroids were resuspended in complete media for further incubation for $24 \mathrm{~h}$ with and without HGX. Finally, the fixation and mounting of coverslips was done by following the same protocol as mentioned in confocal imaging studies section. The spheroids were imaged under $10 \times$ resolution.

\section{ASSOCIATED CONTENT}

\section{S) Supporting Information}

The Supporting Information is available free of charge at https://pubs.acs.org/doi/10.1021/acsbiomaterials.1c01085.

Table S1, sequences of oligonucleotides used for the synthesis of DNA hydrogels; Figure S1, diffusion constant as a function of temperature for different nanostar systems; Figure S2, DNA hydrogels stimulate cell adhesion and enhance cell spreading (PDF)

\section{AUTHOR INFORMATION}

\section{Corresponding Author}

Dhiraj Bhatia - Biological Engineering and Center for Biomedical Engineering, Indian Institute of Technology Gandhinagar, Gandhinagar, Gujarat 382355, India; ○ orcid.org/0000-0002-1478-6417; Email: dhiraj.bhatia@ iitgn.ac.in

\section{Authors}

Shanka Walia - Biological Engineering, Indian Institute of Technology Gandhinagar, Gandhinagar, Gujarat 382355, India

Vinod Morya - Biological Engineering, Indian Institute of Technology Gandhinagar, Gandhinagar, Gujarat 382355, India

Ankit Gangrade - Biological Engineering, Indian Institute of Technology Gandhinagar, Gandhinagar, Gujarat 382355, India

Supriyo Naskar - Center for Condensed Matter Theory, Department of Physics, Indian Institute of Science, Bangalore 560012, India; $\odot$ orcid.org/0000-0002-3690-6483

Aditya Guduru Teja - Chemical Engineering, Indian Institute of Technology Gandhinagar, Gandhinagar, Gujarat 382355, India
Sameer Dalvi - Chemical Engineering and Center for Biomedical Engineering, Indian Institute of Technology Gandhinagar, Gandhinagar, Gujarat 382355, India; - orcid.org/0000-0001-5262-8711

Prabal K Maiti - Center for Condensed Matter Theory, Department of Physics, Indian Institute of Science, Bangalore 560012, India; ㅇo orcid.org/0000-0002-9956-1136

Chinmay Ghoroi - Chemical Engineering, Indian Institute of Technology Gandhinagar, Gandhinagar, Gujarat 382355, India; O orcid.org/0000-0002-2768-5973

Complete contact information is available at:

https://pubs.acs.org/10.1021/acsbiomaterials.1c01085

\section{Author Contributions}

D.B. conceived the idea and planned the experiments. S.W. did the synthesis, characterization by confocal miscroscopy, and cellular area change experiments along with immunofluorescence experiments. V.M. did the characterization by gel electrophoresis, DLS, and AFM imaging of the hydrogels. A.G. did the uptake experiments of $\mathrm{Tf}, \mathrm{Gal} 3$, and CTxB. A.T. did the confocal imaging of hydrogels and 3D spheroid invasion assays. S.N. did the MD simulations of the hydrogels. Professors P.M., C.G., and S.D. provided detailed mentorship at every step and aspect of the manuscript. S.W., V.M., A.G., A.T., and S.N. analyzed all the data and it was cross-checked by all mentors. All the authors contributed to writing and reading the manuscript draft.

\section{Author Contributions}

${ }^{\dagger}$ S.W. and V.M. contributed equally to this work

\section{Notes}

The authors declare no competing financial interest.

\section{ACKNOWLEDGMENTS}

We sincerely thank all the members of D.B.'s group for critically reading the manuscript and their valuable feedback. S.W., V.M., A.G., and A.T. thank IITGN-MHRD, GoI, for postdoctoral and PhD fellowships. S.N. acknowledges an SRF fellowship from CSIR, India. D.B. thanks SERB, GoI, for a Ramanujan Fellowship, IITGN for the startup grant, and DBTEMR, Gujcost-DST, GSBTM, and BRNS-BARC for research grants. The imaging facilities of CIF at IIT Gandhinagar are acknowledged. We sincerely thank IISc Bangalore for the computational support. The work in host laboratories is funded by MHRD and DST-SERB and DBT, GoI.

\section{REFERENCES}

(1) Yue, B. Biology of the Extracellular Matrix: An Overview. J. Glaucoma 2014, 23, S20-S23.

(2) Hinderer, S.; Layland, S. L.; Schenke-Layland, K. ECM and ECM-like Materials - Biomaterials for Applications in Regenerative Medicine and Cancer Therapy. Adv. Drug Delivery Rev. 2016, 97, 260-269.

(3) Finke, A.; Schneider, A.-K.; Spreng, A.-S.; Leist, M.; Niemeyer, C. M.; Marx, A. Functionalized DNA Hydrogels Produced by Polymerase-Catalyzed Incorporation of Non-Natural Nucleotides as a Surface Coating for Cell Culture Applications. Adv. Healthcare Mater. 2019, 8 (9), e1900080.

(4) Antebi, B.; Zhang, Z.; Wang, Y.; Lu, Z.; Chen, X.-D.; Ling, J. Stromal-Cell-Derived Extracellular Matrix Promotes the Proliferation and Retains the Osteogenic Differentiation Capacity of Mesenchymal Stem Cells on Three-Dimensional Scaffolds. Tissue Eng., Part C 2015, 21 (2), 171-181. 
(5) Xu, Y.; Patnaik, S.; Guo, X.; Li, Z.; Lo, W.; Butler, R.; Claude, A.; Liu, Z.; Zhang, G.; Liao, J.; Anderson, P. M.; Guan, J. Cardiac Differentiation of Cardiosphere-Derived Cells in Scaffolds Mimicking Morphology of the Cardiac Extracellular Matrix. Acta Biomater. 2014, 10 (8), 3449-3462.

(6) Pan, J.; Liu, N.; Sun, H.; Xu, F. Preparation and Characterization of Electrospun PLCL/Poloxamer Nanofibers and Dextran/Gelatin Hydrogels for Skin Tissue Engineering. PLoS One 2014, 9 (11), e112885.

(7) Hughes, A. J.; Miyazaki, H.; Coyle, M. C.; Zhang, J.; Laurie, M. T.; Chu, D.; Vavrušová, Z.; Schneider, R. A.; Klein, O. D.; Gartner, Z. J. Engineered Tissue Folding by Mechanical Compaction of the Mesenchyme. Dev. Cell 2018, 44 (2), 165-178.

(8) Shu, X. Z.; Ghosh, K.; Liu, Y.; Palumbo, F. S.; Luo, Y.; Clark, R. A.; Prestwich, G. D. Attachment and Spreading of Fibroblasts on an RGD Peptide-Modified Injectable Hyaluronan Hydrogel. J. Biomed. Mater. Res. 2004, 68 (2), 365-375.

(9) Bracaglia, L. G.; Fisher, J. P. Extracellular Matrix-Based Biohybrid Materials for Engineering Compliant, Matrix-Dense Tissues. Adv. Healthcare Mater. 2015, 4 (16), 2475-2487.

(10) Ramadhan, W.; Kagawa, G.; Moriyama, K.; Wakabayashi, R.; Minamihata, K.; Goto, M.; Kamiya, N. Construction of Higher-Order Cellular Microstructures by a Self-Wrapping Co-Culture Strategy Using a Redox-Responsive Hydrogel. Sci. Rep. 2020, 10 (1), 6710.

(11) Geckil, H.; Xu, F.; Zhang, X.; Moon, S.; Demirci, U. Engineering Hydrogels as Extracellular Matrix Mimics. Nanomedicine (London, U. K.) 2010, 5 (3), 469-484.

(12) Zhong, G.; Yao, J.; Huang, X.; Luo, Y.; Wang, M.; Han, J.; Chen, F.; Yu, Y. Injectable ECM Hydrogel for Delivery of BMSCs Enabled Full-Thickness Meniscus Repair in an Orthotopic Rat Model. Bioactive Materials 2020, 5 (4), 871-879.

(13) Wang, D.; Hu, Y.; Liu, P.; Luo, D. Bioresponsive DNA Hydrogels: Beyond the Conventional Stimuli Responsiveness. Acc. Chem. Res. 2017, 50 (4), 733-739.

(14) Chen, J.; Zhu, Y.; Liu, H.; Wang, L. Tailoring DNA SelfAssembly to Build Hydrogels. Top Curr. Chem. (Cham) 2020, 378 (2), 32.

(15) Sharma, A.; Sharma, P.; Roy, S. Elastin-Inspired Supramolecular Hydrogels: A Multifaceted Extracellular Matrix Protein in Biomedical Engineering. Soft Matter 2021, 17 (12), 3266-3290.

(16) Black, L. D.; Allen, P. G.; Morris, S. M.; Stone, P. J.; Suki, B. Mechanical and Failure Properties of Extracellular Matrix Sheets as a Function of Structural Protein Composition. Biophys. J. 2008, 94 (5), 1916-1929.

(17) Watson, J. D.; Crick, F. H. Molecular Structure of Nucleic Acids: A Structure for Deoxyribose Nucleic Acid. Am. J. Psychiatry 2003, 160 (4), 623-624.

(18) Seeman, N. C. Nucleic Acid Junctions and Lattices. J. Theor. Biol. 1982, 99 (2), 237-247.

(19) Morya, V.; Walia, S.; Mandal, B. B.; Ghoroi, C.; Bhatia, D. Functional DNA Based Hydrogels: Development, Properties and Biological Applications. ACS Biomater. Sci. Eng. 2020, 6 (11), 60216035

(20) Xiao, M.; Lai, W.; Wang, X.; Qu, X.; Li, L.; Pei, H. DNA Mediated Self-Assembly of Multicellular Microtissues. Microphysiological Systems 2018, 1 (1), 1.

(21) Um, S. H.; Lee, J. B.; Park, N.; Kwon, S. Y.; Umbach, C. C.; Luo, D. Enzyme-Catalysed Assembly of DNA Hydrogel. Nat. Mater. 2006, 5 (10), 797-801.

(22) Xing, Y.; Cheng, E.; Yang, Y.; Chen, P.; Zhang, T.; Sun, Y.; Yang, Z.; Liu, D. Self-Assembled DNA Hydrogels with Designable Thermal and Enzymatic Responsiveness. Adv. Mater. 2011, 23 (9), $1117-1121$.

(23) Lee, J. B.; Peng, S.; Yang, D.; Roh, Y. H.; Funabashi, H.; Park, N.; Rice, E. J.; Chen, L.; Long, R.; Wu, M.; Luo, D. A Mechanical Metamaterial Made from a DNA Hydrogel. Nat. Nanotechnol. 2012, 7 (12), 816-820.
(24) Xue, H.; Ding, F.; Zhang, J.; Guo, Y.; Gao, X.; Feng, J.; Zhu, X.; Zhang, C. DNA Tetrahedron-Based Nanogels for SiRNA Delivery and Gene Silencing. Chem. Commun. 2019, 55 (29), 4222-4225.

(25) Xing, Z.; Ness, C.; Frenkel, D.; Eiser, E. Structural and Linear Elastic Properties of DNA Hydrogels by Coarse-Grained Simulation. Macromolecules 2019, 52 (2), 504-512.

(26) Khalili, A. A.; Ahmad, M. R. A Review of Cell Adhesion Studies for Biomedical and Biological Applications. Int. J. Mol. Sci. 2015, 16 (8), 18149-18184.

(27) Tan, J.; Gemeinhart, R. A.; Ma, M.; Mark Saltzman, W. Improved Cell Adhesion and Proliferation on Synthetic Phosphonic Acid-Containing Hydrogels. Biomaterials 2005, 26 (17), 3663-3671.

(28) Chen, N.; Zhang, Z.; Soontornworajit, B.; Zhou, J.; Wang, Y. Cell Adhesion on an Artificial Extracellular Matrix Using AptamerFunctionalized PEG Hydrogels. Biomaterials 2012, 33 (5), 13531362

(29) Wang, S.; Sarwat, M.; Wang, P.; Surrao, D. C.; Harkin, D. G.; St John, J. A.; Bolle, E. C. L.; Forget, A.; Dalton, P. D.; Dargaville, T. R. Hydrogels with Cell Adhesion Peptide-Decorated Channel Walls for Cell Guidance. Macromol. Rapid Commun. 2020, 41 (15), 2000295.

(30) Tang, D. D.; Gerlach, B. D. The Roles and Regulation of the Actin Cytoskeleton, Intermediate Filaments and Microtubules in Smooth Muscle Cell Migration. Respir. Res. 2017, 18 (1), 54.

(31) Cantor, J. M.; Ginsberg, M. H. CD98 at the Crossroads of Adaptive Immunity and Cancer. J. Cell Sci. 2012, 125 (6), 13731382

(32) Iacono, K. T.; Brown, A. L.; Greene, M. I.; Saouaf, S. J. CD147 Immunoglobulin Superfamily Receptor Function and Role in Pathology. Exp. Mol. Pathol. 2007, 83 (3), 283-295.

(33) Wu, B.; Cui, J.; Yang, X.-M.; Liu, Z.-Y.; Song, F.; Li, L.; Jiang, J.-L.; Chen, Z.-N. Cytoplasmic Fragment of CD147 Generated by Regulated Intramembrane Proteolysis Contributes to HCC by Promoting Autophagy. Cell Death Dis. 2017, 8 (7), e2925.

(34) Scaltriti, M.; Baselga, J. The Epidermal Growth Factor Receptor Pathway: A Model for Targeted Therapy. Clin. Cancer Res. 2006, 12 (18), 5268-5272.

(35) McMahon, H. T.; Boucrot, E. Molecular Mechanism and Physiological Functions of Clathrin-Mediated Endocytosis. Nat. Rev. Mol. Cell Biol. 2011, 12 (8), 517-533.

(36) Hivare, P.; Rajwar, A.; Gupta, S.; Bhatia, D. Spatiotemporal Dynamics of Endocytic Pathways Adapted by Small DNA Nanocages in Model Neuroblastoma Cell-Derived Differentiated Neurons. ACS Appl. Bio Mater. 2021, 4 (4), 3350-3359.

(37) Chinnapen, D. J.-F.; Chinnapen, H.; Saslowsky, D.; Lencer, W. I. Rafting with Cholera Toxin: Endocytosis and Tra/Cking from Plasma Membrane to ER. FEMS Microbiol. Lett. 2007, 266 (2), 129137.

(38) Costa, E. C.; Moreira, A. F.; de Melo-Diogo, D.; Gaspar, V. M.; Carvalho, M. P.; Correia, I. J. 3D Tumor Spheroids: An Overview on the Tools and Techniques Used for Their Analysis. Biotechnol. Adv. 2016, 34 (8), 1427-1441.

(39) Brüningk, S. C.; Rivens, I.; Box, C.; Oelfke, U.; ter Haar, G. 3D Tumour Spheroids for the Prediction of the Effects of Radiation and Hyperthermia Treatments. Sci. Rep. 2020, 10, 1653.

(40) Xing, Z.; Caciagli, A.; Cao, T.; Stoev, I.; Zupkauskas, M.; O’Neill, T.; Wenzel, T.; Lamboll, R.; Liu, D.; Eiser, E. Microrheology of DNA Hydrogels. Proc. Natl. Acad. Sci. U. S. A. 2018, 115 (32), $8137-8142$

(41) Foty, R. A Simple Hanging Drop Cell Culture Protocol for Generation of 3D Spheroids. J. Visualized Exp. 2011, 51 (No), 2720. 